\title{
Biocompatibility and Bone Formation of Flexible, Cotton Wool-like PLGA/Calcium Phosphate Nanocomposites in Sheep
}

\author{
Oliver D. Schneider ${ }^{1}$, Dirk Mohn ${ }^{1}$, Roland Fuhrer ${ }^{1}$, Karina Klein ${ }^{2}$, Käthi Kämpf ${ }^{2}$, Katja M.R. Nuss ${ }^{2}$, \\ Michèle Sidler ${ }^{2}$, Katalin Zlinszky ${ }^{2}$, Brigitte von Rechenberg ${ }^{2,3}$ and Wendelin J. Stark ${ }^{*}, 1,3$
}

\author{
${ }^{1}$ Institute for Chemical and Bioengineering, Department of Chemistry and Applied Biosciences, ETH Zurich, 8093 \\ Zurich, Switzerland \\ ${ }^{2}$ Musculoskeletal Research Unit (MSRU), Equine Hospital, University of Zurich, 8057 Zurich, Switzerland \\ ${ }^{3}$ Center of Applied Biotechnology and Molecular Medicine (CABMM), Equine Hospital, Vetsuisse Faculty, 8057 Zurich, \\ Switzerland
}

\begin{abstract}
Background: The purpose of this preliminary study was to assess the in vivo performance of synthetic, cotton wool-like nanocomposites consisting of a biodegradable poly(lactide-co-glycolide) fibrous matrix and containing either calcium phosphate nanoparticles (PLGA/CaP 60:40) or silver doped CaP nanoparticles (PLGA/Ag-CaP 60:40). Besides its extraordinary in vitro bioactivity the latter biomaterial $(0.4 \mathrm{wt} \%$ total silver concentration $)$ provides additional antimicrobial properties for treating bone defects exposed to microorganisms.

Materials and Methods: Both flexible artificial bone substitutes were implanted into totally 16 epiphyseal and metaphyseal drill hole defects of long bone in sheep and followed for 8 weeks. Histological and histomorphological analyses were conducted to evaluate the biocompatibility and bone formation applying a score system. The influence of silver on the in vivo performance was further investigated.

Results: Semi-quantitative evaluation of histology sections showed for both implant materials an excellent biocompatibility and bone healing with no resorption in the adjacent bone. No signs of inflammation were detectable, either macroscopically or microscopically, as was evident in $5 \mu \mathrm{m}$ plastic sections by the minimal amount of inflammatory cells. The fibrous biomaterials enabled bone formation directly in the centre of the former defect. The area fraction of new bone formation as determined histomorphometrically after 8 weeks implantation was very similar with $20.5 \pm 11.2 \%$ and $22.5 \pm 9.2 \%$ for PLGA/CaP and PLGA/Ag-CaP, respectively.

Conclusions: The cotton wool-like bone substitute material is easily applicable, biocompatible and might be beneficial in minimal invasive surgery for treating bone defects.
\end{abstract}

Keywords: In vivo, bone regeneration, flexible, scaffold, silver.

\section{INTRODUCTION}

Every year, more than one million bone grafts are needed worldwide. The golden standard for orthopaedic cure is still auto- and allografting [1, 2]. However, this method suffers from limited graft availability, the requirement of a second surgery and a serious risk of pain and infection at the donorsite [3-5]. Therefore, synthetic bone replacements are an attractive alternative, and should enable fast bone regeneration and show good handling properties during surgical application, especially in minimally invasive surgery. Additionally, a synthetic biomaterial should be bioresorbable and ideally exhibit antimicrobial properties to counteract postoperative infections. In this regard, silver is known for thousands of years for its antimicrobial properties with low toxicity to human cells $[6,7]$ and has been applied in wound

*Address correspondence to this author at the ETH Zurich, Institute for Chemical and Bioengineering, Department of Chemistry and Applied Biosciences, Wolfgang-Pauli-Str. 10, HCI E 107, 8093 Zurich, Switzerland; Tel: +41 4463209 80; Fax: +41 4463310 83;

E-mail:wendelin.stark@chem.ethz.ch dressings [8] or in bone cements [9]. In terms of bioactivity, specific materials such as tricalcium phosphate (TCP) or bioactive glasses are synthetic materials clinically used in orthopaedics [10-12] in the form of blocks, cements or granules. They are osteoconductive, enhance osteoblast differentiation and can induce strong bonding to bone.

However, surgery is often complicated by the brittleness (of granules) and the lack of possibility to shape the implant material during the operation. Flexibility can be obtained by incorporating ceramics as fillers in biodegradable polymers where it may improve mechanical properties and processability [13, 14]. Poly(lactide-co-glycolide) (PLGA) is a hydrolytically degradable and biocompatible polymer [15] and has been approved by the Food and Drug Administration (FDA) for drug delivery and in nerve- and bone regeneration. Electrospinning has turned out as a suitable method to create elastic, open structured, highly porous networks of polymer fibres [16, 17] which enable cell attachment, cell migration and nutrient transport [18] in a scaffold. It has been recently shown that an electrospun 
biodegradable matrix can improve the regeneration of dense cortical bone in vivo [19].

By using very fine powders (nanoparticles), a nanostructured surface can be induced in the scaffold and thus mimic the structure of natural bone with a significant enhancement of osteoblast activity [20-23] and protein adsorption [24]. Calcium phosphate ( $\mathrm{CaP})$ nanoparticles, in their amorphous state, can serve as a precursor for rapid hydroxyapatite formation $[25,26]$ inside the body just hours after implantation, as shown for films [27] and bone screws [28] where the mineral improved degradation and mechanical properties. We have recently prepared flexible PLGA/amorphous-CaP nanocomposites with a cotton woollike appearance (bone wool) and found efficient osteogenic differentiation of human mesenchymal stem cells [29], easy applicability and proper bone formation in calvarial defects in rabbits [30]. Optional silver doping resulted in enhanced antimicrobial properties against Escherichia coli [31] when compared to tetracycline controls.

The objective in the present study is a pre-clinical evaluation of such cotton wool-like scaffolds in non-load bearing bone defects (e.g. socket preservation; osteoporosis; sinus lift, etc). More specifically, we tested the in vivo performance using a drill hole defect model in the metaphysis and epiphysis of long bone in sheep following well established procedures [32]. The effect of silver on the biocompatibility and cellular reactions during bone regeneration was assessed through qualitative and semiquantitative histology after eight weeks implantation. Histomorphometrical analysis was performed to evaluate new bone formation and compared to earlier studies [33, 34] with the same drill hole defects left open (controls, no treatment) or filled with $\mathrm{CaP}$ cements or porous $\beta$-TCP blocks (recently developed, modern treatments).

\section{MATERIALS AND METHODS}

\subsection{Cotton Wool-like PLGA/CaP and PLGA/Ag-CaP Nanocomposites}

$\mathrm{X}$-ray diffraction (XRD)-amorphous calcium phosphate $\left(\mathrm{CaP}, \mathrm{Ca}_{3}\left(\mathrm{PO}_{4}\right)_{2}\right)$ and silver calcium phosphate nanoparticles (Ag-CaP, 1 wt $\%$ Ag on $\mathrm{CaP}$ ) were prepared by flame spray synthesis [35-38] and thoroughly characterized (see Suppl. material 1). Clinically approved poly(lactide-co-glycolide) (PLGA) was purchased from Boehringer Ingelheim with a copolymer ratio of 85:15 (Resomer® Sample MD Type RG) and with a weight and number average molecular weight of $305^{\prime} 440 \mathrm{~g} / \mathrm{mol}$ and $110^{\prime} 500 \mathrm{~g} / \mathrm{mol}$, respectively. PLGA/CaP 60:40 and PLGA/Ag-CaP 60:40 (total silver concentration in scaffold $=0.4 \mathrm{wt} \%$ ) scaffolds were fabricated by lowtemperature electrospinning [17] (see Suppl. material 1), portioned (109-134 mg) and UV-sterilized $\left(50 \mathrm{~W} / \mathrm{m}^{2}, 90\right.$ $\min )$.

\subsection{Animal Model in Sheep}

Two female, white Swiss Alpine sheep with an age of 2.5 years and a bodyweight of 43-46 kg served as experimental animals (sheep 3118 and sheep 3119). The animal study was conducted according to the Swiss Law of animal protection and welfare and was permitted through the official, Swiss authorities (permission \# 74/2008). Prior to anaesthesia, the health status of the animals was checked. A standardized balanced anaesthetic protocol was used. After $24 \mathrm{~h}$ of fasting, premedication and sedation was achieved (Buprenorphine (Temgesic ${ }^{\circledR}, 0.01 \mathrm{mg} / \mathrm{kg}$ im, Essex Chemie AG, Switzerland); Xylazine (Rompun ${ }^{\circledR} 2 \%, 0.1 \mathrm{mg} / \mathrm{kg} \mathrm{im}$, Provet AG, Switzerland)). Anaesthesia was induced (Diazepam (Valium ${ }^{\circledR}, 0.1 \mathrm{mg} / \mathrm{kg}$ iv, Roche Pharma AG, Switzerland); Ketamine (Narketan $10^{\circledR}, 4 \mathrm{mg} / \mathrm{kg}$ iv, Vetoquinol AG, Switzerland); Propofol (Propofol ${ }^{\circledR}, 0.4$ $\mathrm{mg} / \mathrm{kg}$ iv, Fresenius Kabi, Switzerland)) before general inhalation. Anaesthesia was maintained using isoflurane in $100 \%$ oxygen via an appropriately sized breathing system and a constant rate infusion of Propofol (Propofol ${ }^{\circledR}$, $0.1 \mathrm{mg} / \mathrm{kg} / \mathrm{h}$, Fresenius Kabi, Switzerland). Equine tetanus serum (Tetanus serum 3000 IU, Veternaria AG, Switzerland), Benzylpenicillin (Procain-Penicillin Streuli ad us. vet., 30'000 IU/kg, G. Streuli \& Co AG; Switzerland), Gentamicin (Vetagent ${ }^{\circledR}$ ad us. vet., $6 \mathrm{mg} / \mathrm{kg}$, Veterinaria AG, Switzerland) and Carprofen (Rimadyl ${ }^{\circledR}, 4 \mathrm{mg} / \mathrm{kg}$, Pfizer AG, Switzerland) were given prophylactically for 3 days to avoid peri- and postoperative infection, pain and acute inflammation.

The sheep were placed in left or right lateral recumbency on the operating table. Routinely, a lateral surgical approach to the bone was used. Totally 8 drill hole defects (diameter $=$ $8 \mathrm{~mm}$, depth $=13 \mathrm{~mm}$ ) were created with a drill bit in the distal (di) epiphysis and proximal (pr) metaphysis of the right and left humerus (hu) and femur (fe) as described in detail by Nuss et al. [32]. The bone cavities were carefully washed to eliminate bone debris and dried before filling them with PLGA/CaP and PLGA/Ag-CaP scaffolds ( 109$134 \mathrm{mg}$ ). The wound was closed and the skin was stapled. After recovery the sheep were kept in stalls with free access to food and water. The sheep were sacrificed at 8 weeks after surgery.

\subsection{Macroscopic and Radiographic Analysis}

The bone samples were harvested directly after slaughter of sheep. The defects were macroscopically evaluated with respect to filling of the defect with bone, respectively fibrous tissue and signs of inflammation in the adjacent tissue. For detailed radiographs, bone specimens were cut perpendicular to the bone defect using a band saw, and bone blocks were documented with a Faxitron $(55 \mathrm{kV}, 5 \mathrm{~s}$, Faxitron Cabinet Xray system, Model MX-20). Contact microradiographs (400 $\mu \mathrm{m})$ (Faxitron, X-ray System) were created with a high resolution film (X-OMAT MA film, Kodak Film, France) and used to compare with histological grinding sections.

\subsection{Histology}

Histology sections of extracted bone samples were prepared as described by Apelt et al. [33]. Briefly, the drill hole defects were fixed in $4 \%$ paraformaldehyde for 2 weeks, dehydrated in increasing grades of ethanol (50-100\%) before defatting with xylene. Next, embedding was performed by placing the bone samples in acrylic resin (Histo-Dur ${ }^{\circledR}$, Leica, Switzerland). Transverse sections were made from the polymerized blocks in the centre and at a $90^{\circ}$ angle to the former drill hole defects. Grinding sections (30$40 \mu \mathrm{m})$ were surface stained with toluidine blue, while thin sections $(5 \mu \mathrm{m})$ were stained with toluidine blue and van Kossa/Mc Neal's Tetrachrome. 


\subsection{Histomorphometry}

The bone regeneration was quantitatively evaluated in the former drill hole defect area. Briefly, the grinding sections were read in a computer program (Leica IM 1000, Leica Microsystems, Switzerland) at a magnification of 12 by means of a macroscope (Leica ${ }^{\mathrm{TM}}$ M420, Leica Microsystems) equipped with a digital camera (Leica ${ }^{\mathrm{TM}}$ DFC 320, Leica Microsytems). The margins of the cylindrical defects (diameter $=8 \mathrm{~mm}$ ) were identified on the digital images. Then, the bone tissue within the defect area was marked (pixels) manually using commercial software (Adobe Photoshop) and a standardized computer macro was applied (Quips, QWin, Leica Microsystems) to detect areas of new bone formation. The percentage area fraction of new bone formation was calculated from the marked pixels and the total pixel number of the defect.

\subsection{Qualitative and Semi-Quantitative Evaluation}

Thin histological sections were investigated with a microscope (DMR, Leica Microsystems) for semiquantitative and qualitative evaluation. The quality of new bone formation, resorption of bone cotton-wool and/or adjacent bone was assessed. The influences of silver containing material as well as signs of inflammation were indirectly recorded, such that cellular reactions were studied. A score system was applied to evaluate semi-quantitatively the cellular reactions in three different zones from the border to the centre of the former defect at a magnification of $400 \mathrm{x}$ (see Suppl. material 2). Macrophages, fibroblasts/fibrocytes and lymphocytes/plasma cells were assessed according to the percentage fraction of the cells per power field (score: 0 for $0 \%, 1$ for $1-25 \%, 2$ for $26-50 \%, 3$ for $>50 \%$ ). Osteoclasts, foreign body giant cells (FBGC), circles of macrophages (macrophages arranged in circles around biomaterial but have not yet fused to FBGC) and bone islands (small areas of newly formed bone) were counted per power field (score: 0 for none, 1 for 1-5, 2 for 6-10, 3 for > 11).

\subsection{Statistical Analysis}

Statistical analysis using student t-test for independent values was carried out on histomorphometry (bone formation) and on the semi-quantitative (cell number, type and location) evaluation. The effect of silver on biocompatibility and bone formation was investigated in the two groups PLGA/CaP and PLGA/Ag-CaP. Results were considered to be significant at $\mathrm{p}<0.05$.

\section{RESULTS}

\subsection{CaP and Ag-CaP nanoparticle preparation}

$\mathrm{CaP}$ nanoparticles had a size of $20-50 \mathrm{~nm}$ and were optionally doped with silver (Ag-CaP) (Fig. 1a). The specific surface area of the $\mathrm{CaP}$ and $\mathrm{Ag}-\mathrm{CaP}$ nanoparticles was $79( \pm$ $3 \%) \mathrm{m}^{2} / \mathrm{g}$ and $73( \pm 3 \%) \mathrm{m}^{2} / \mathrm{g}$, respectively. The amorphous state of the as-prepared nanoparticles was confirmed by $\mathrm{X}$ ray diffraction (XRD) patterns showing a broad characteristic peak at a $2 \theta$ value of $31.0^{\circ}$ (see Suppl. material $1)$. Distinct patterns characteristic for crystalline $\beta$-TCP were obtained after sintering at $900^{\circ} \mathrm{C}$ for $30 \mathrm{~min}$ and confirmed the chemically correct composition. The high phase purity of the nanoparticles was corroborated by FTIR-spectroscopy (see Suppl. material 1). Sintered powder showed characteristic peaks for $\beta$-TCP whereas peaks for impurities such as pyrophosphate or IR peaks ascribed to OH-groups such as they exist in hydroxyapatite were not detected. The silver content in the $\mathrm{Ag}-\mathrm{CaP}$ nanoparticles measured by atom absorption spectroscopy was $0.95 \mathrm{wt} \%$ and confirmed the reliability of the flame process in terms of silver incorporation.

\subsection{Bioactive, Flexible Nanocomposites (Bone Wool)}

Scaffolds containing $40 \mathrm{wt} \% \mathrm{CaP}$ or $\mathrm{Ag}-\mathrm{CaP}$ in PLGA were prepared by low-temperature electrospinning. The aselectrospun scaffolds could be manually transformed into a soft and compressible biomaterial with a cotton wool-like appearance (Fig. 1b). This flexible material allowed easy portioning and plugging in even complex shaped bone defects. The as-electrospun three-dimensional network consisted of homogenous fibres within a diameter range of $5-10 \mu \mathrm{m}$ as shown by scanning electron microscopy. The fibres themselves were nanostructured due to the incorporated nanoparticles (Fig. 1c). The specific surface area of both fibrous scaffolds was $2( \pm 3 \%) \mathrm{m}^{2} / \mathrm{g}$.
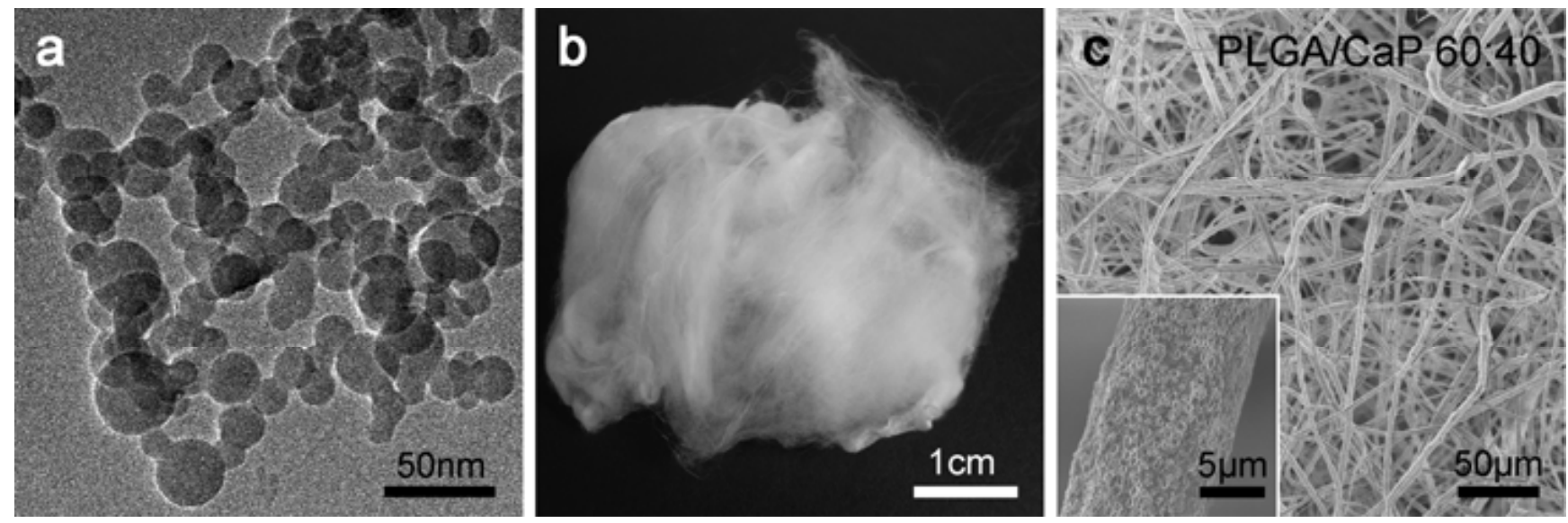

Fig. (1). Characterisation of the materials. (a) Transmission electron microscopic image of as-prepared calcium phosphate nanoparticles. (b) Scaffolds in uncompressed form have a cotton wool-like appearance. (c) Scanning electron microscopic image of low-temperature electrospun PLGA/CaP 60:40 fibres showing a nanostructured surface (inlet). 

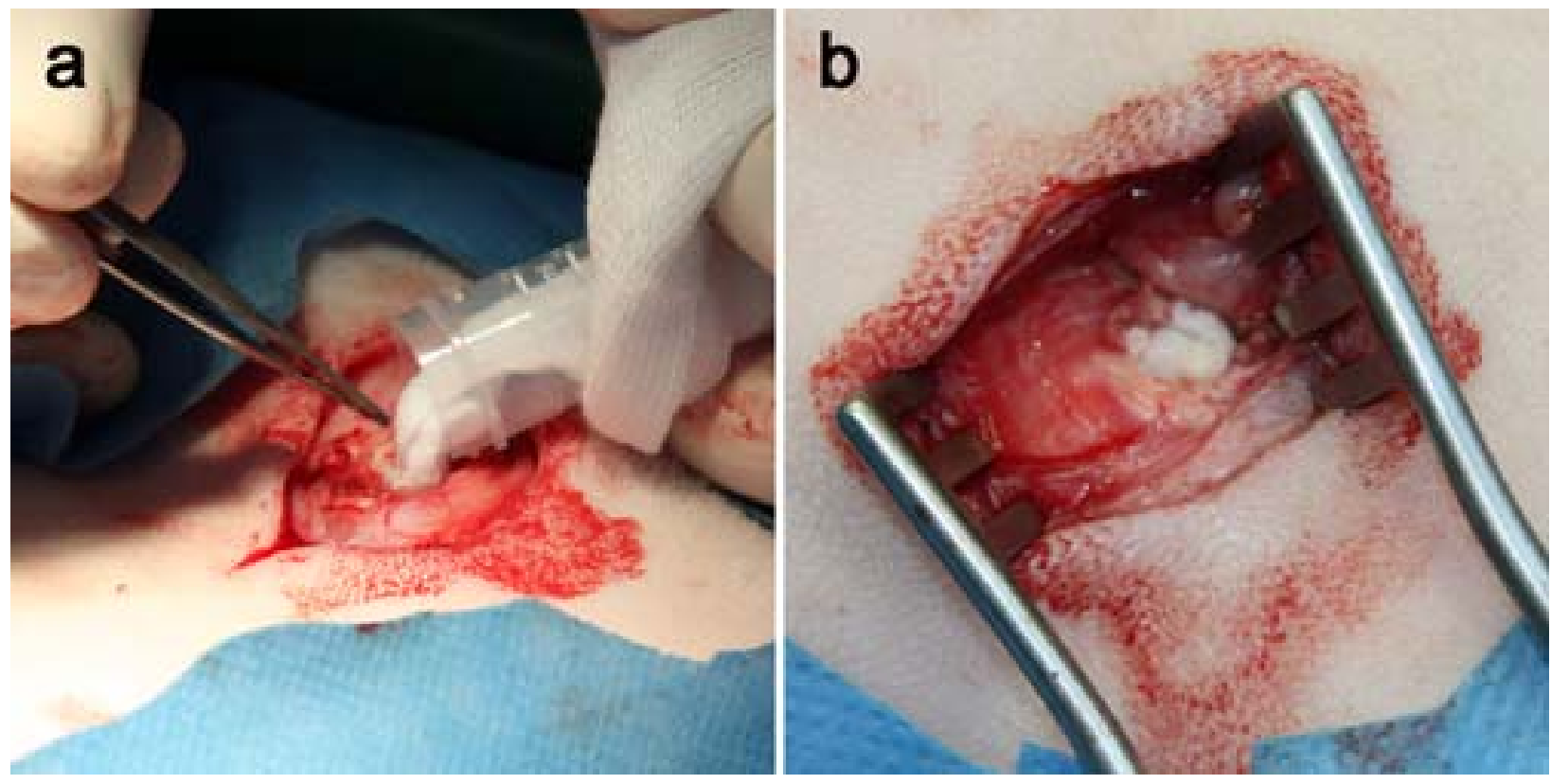

Fig. (2). Surgical procedure. (a, b) Filling of the drill hole defects (diameter $=8 \mathrm{~mm}$, depth $=13 \mathrm{~mm}$ ) in white Swiss Alpine sheep. The lowtemperature electrospun PLGA/CaP and PLGA/Ag-CaP scaffolds can be easily applied.

\subsection{Surgery in Sheep}

The surgery of the two sheep was performed without complications. The handling of the electrospun PLGA/CaP and PLGA/Ag-CaP nanocomposites was straightforward and could be easily placed in the created drill hole defects (Fig. 2). The packing density $\left(180 \mathrm{mg} / \mathrm{cm}^{3}\right)$ of the fibrous material was kept the same as in an earlier study in calvarial defects in rabbits [30]. Since this bone cotton wool is soft and flexible, there was no risk of damaging tissue when applying this artificial bone graft. Further, a proper adaptation to the bone defect was reached with this compressible material, which was also easily wetted with blood. Recovery from surgery went well for all sheep and wound healing was without complication, when skin staples were removed at 10 days after surgery.

\subsection{Macroscopic and Radiographic Analysis}

The scaffolds were well integrated in the original bone defects and covered with a layer of soft tissue at eight weeks after implantation. No signs of inflammatory reactions, such as necrosis or reddening suggesting implant rejection were found macroscopically. All originally created drill hole defects could be located radiographically except for one defect (humerus left proximal, filled with PLGA/CaP), which could not be found anymore. Microradiographs (see Suppl. material 3) showed bone formation inside the original defect and no signs of sclerosis within the adjacent bone. Calcification and new bone formation were in agreement with the ground sections, which were considered more appropriate to be used for quantitative evaluation of bone formation.

\subsection{Histomorphometry}

The quantitative evaluation of bone formation was performed on the ground histological sections after surface staining with toluidine blue (Fig. 3). New bone formation (coloured in blue) was possible everywhere in the created circular defects. Since a clear differentiation between the remaining fibrous biomaterial and the granulation tissue was not possible, only the bone matrix was coloured in the original defect. The area fraction of newly formed bone for both materials is independent of the locations of the defect (Fig. 4). The average area of newly formed bone amounted to $20.5 \pm 11.2 \%$ for PLGA/CaP and $22.5 \pm 9.2 \%$ for PLGA/Ag-CaP treated defects. There was no statistically significant difference $(p>0.05)$ in bone formation for these two groups. The ratio between granulation tissue and remaining biomaterials was around 80:20 at two months after insertion of the implants.

\subsection{Qualitative and Semi-Quantitative Evaluation}

The histological thin sections confirmed the biocompatibility of the bone substitutes and appeared the same for both electrospun scaffolds (Fig. 5). Both biomaterials were biocompatible and were mostly resorbed by macrophages, which were typically arranged as circles around the material (Fig. 5c). Bone islands were generally formed in regions where circles of macrophages were previously present (Fig. 5b). The cytoplasm of macrophages was loaded with material indicated through the typical birefringent appearance of polymers. The resorption zone was rich in cells and dominated by macrophages and osteoprogenitor cells (Fig. 5f). The active osteoid producing osteoblasts were not counted because they were abundant and always easily identified at the surface of the osteoid. At the investigated time period of eight weeks, only a few osteoclasts appeared and were found mostly at the calcified bone surface at the defect margins starting to remodel the newly formed woven bone. Blood vessels and erythrocytes were evenly distributed and followed the ossification front. No statistically significant differences could be found 

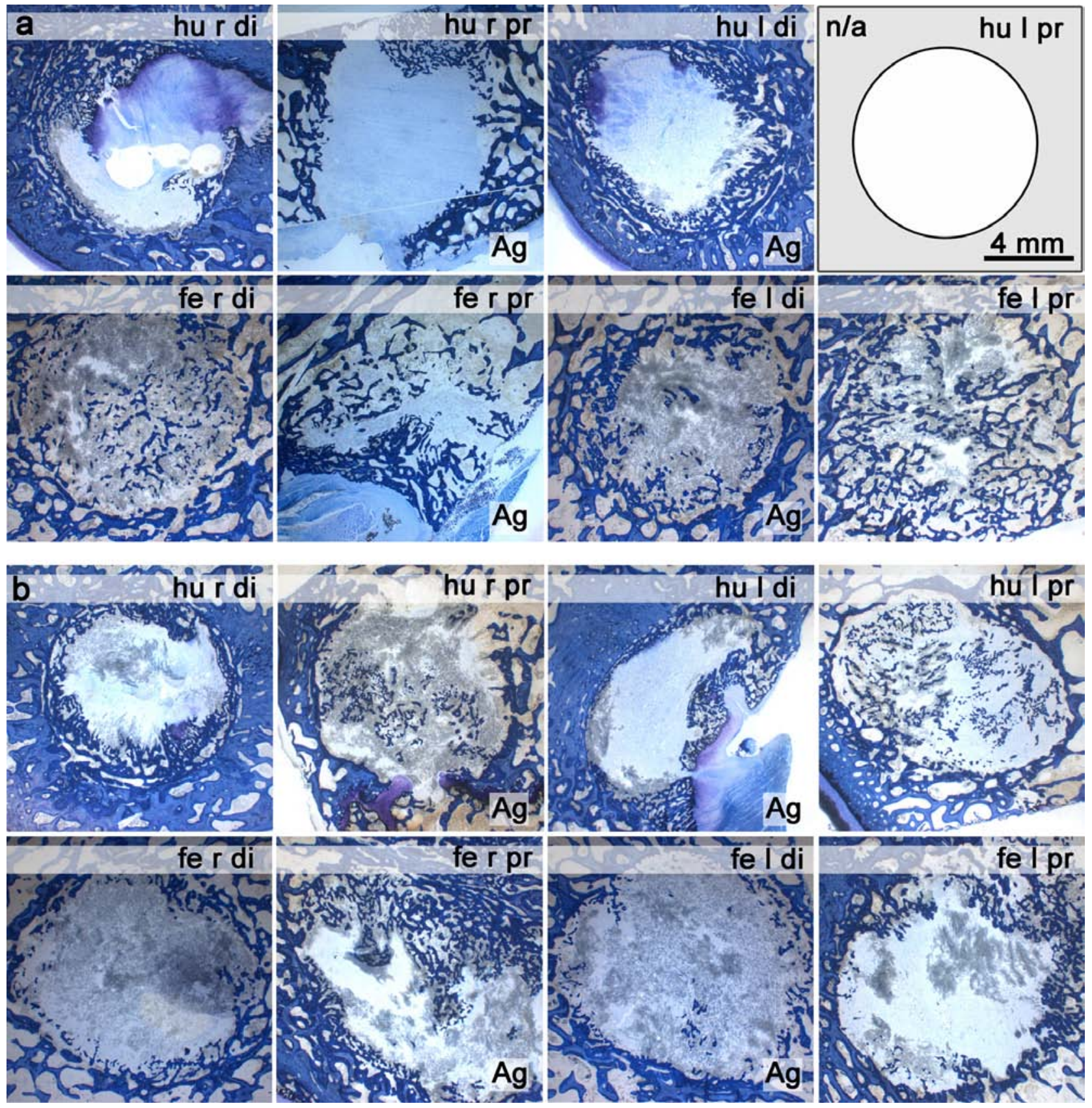

Fig. (3). Histological ground sections (surface stained with toluidine blue) after 8 weeks implantation of PLGA/CaP $60: 40$ ( $1^{\text {st }}$ and $4^{\text {th }}$ column) and PLGA/Ag-CaP 60:40 ( $2^{\text {nd }}$ and $3^{\text {rd }}$ column) nanocomposites in (a) sheep 3118 and (b) sheep 3119. Formation of new bone (blue) is visible in the drill hole defects (diameter $=8 \mathrm{~mm}$, depth $=13 \mathrm{~mm}$ ). The ground section of sheep 3118 humerus left proximal is not available.

between the two groups PLGA/CaP 60:40 and PLGA/AgCaP 60:40 regarding bone remodeling and cell types (Table 1, Suppl. material 4) after eight weeks implantation. Fibroblasts and fibrocytes were found only in one defect with a percentage larger than $1 \%$ per power field. Overall, the presence of foreign body giant cells was low (1-5) and independent of location and group. Lymphocytes and plasma cells were generally low and equally distributed over the area, mostly close to vessels. They never appeared in clusters close to the material. Their distribution was similar as found in physiologic bone healing.

\section{DISCUSSION}

The present in vivo performance of bone wool is based on earlier in vitro/in vivo studies [29, 30] optionally showing antimicrobial properties [31]. The here used protocol for biocompatibility and bone formation using drill hole defects in humerus and femur of Swiss Alpine sheep has been well established [32-34] since sheep are suitable for orthopaedic research due to a bone structure and metabolism comparable to humans [39]. Evaluation after eight weeks allows following cellular (bone formation) and inflammatory reactions. 


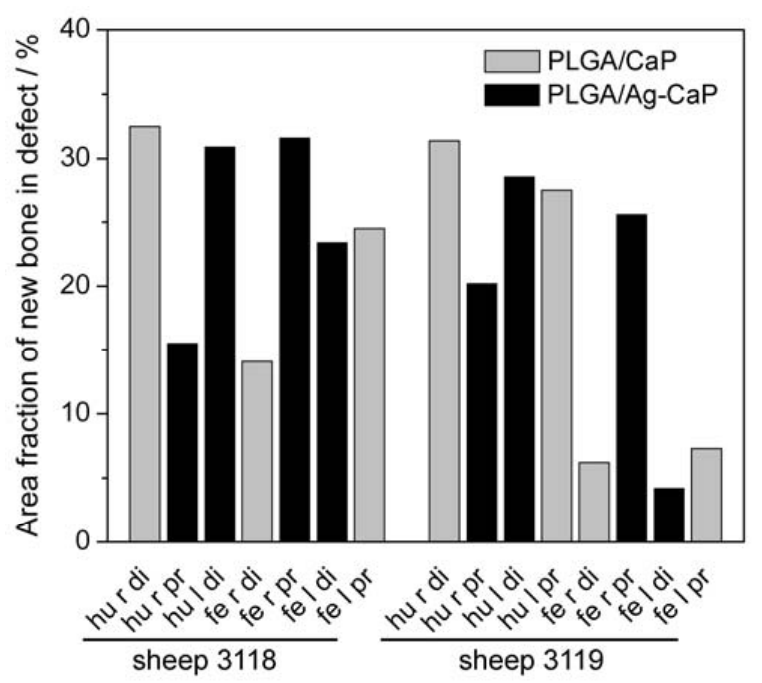

Fig. (4). Area fraction of newly formed bone within the original drill hole defect as a percentage of the total defect area. PLGA/CaP and PLGA/Ag-CaP scaffolds were implanted for eight weeks in distal epiphysis (di) and proximal metaphysis (pr) of the right (r) and left (l) humerus (hu) and femur (fe).
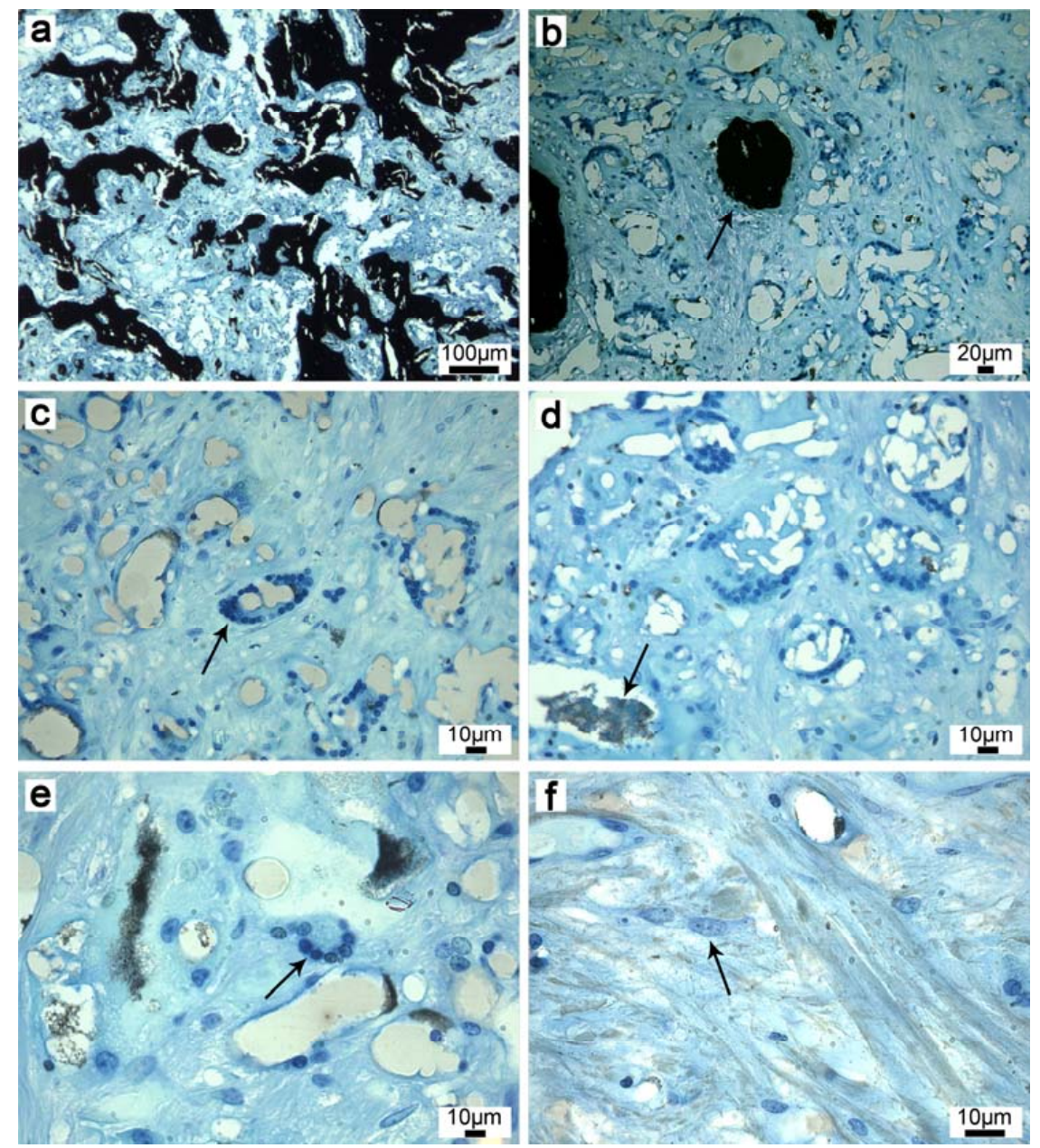

Fig. (5). Histological thin sections after 8 weeks implantation (representative for both nanocomposites, surface stained with van Kossa). (a, b) New bone is formed within the resorption zone (arrow). (c) Macrophages arranged in circles resorb the (d) biomaterial and (e) fuse finally to foreign body giant cells. The resorption zone consists further of (f) osteoprogenitor cells. The absence of clusters of lymphocytes, plasma cells and fibrocytes confirmed the biocompatibility of the electrospun PLGA/CaP and PLGA/Ag-CaP nanocomposites. 
Table 1. Semi-Quantitative Analysis of Biocompatibility and Bone Formation After 8 Weeks Implantation Applying a Score System in Three Different Zones from the Border Towards Centre of the Former Defect

\begin{tabular}{|c|c|c|c|c|c|}
\hline Variable & Group & Zone 1 & Zone 2 & Zone 3 & Total \\
\hline \hline Lymphocytes/plasma cells $^{\mathrm{a}}$ & PLGA/CaP & $0.3 \pm 0.5$ & $0.3 \pm 0.5$ & $0.1 \pm 0.4$ & $0.7 \pm 0.5$ \\
& PLGA/Ag-CaP & $0.3 \pm 0.5$ & $0.1 \pm 0.4$ & $0.0 \pm 0.0$ & $0.4 \pm 0.7$ \\
\hline Macrophages $^{\mathrm{a}}$ & PLGA/CaP & $1.0 \pm 0.0$ & $1.1 \pm 0.4$ & $1.1 \pm 0.4$ & $3.3 \pm 0.8$ \\
& PLGA/Ag-CaP & $1.1 \pm 0.4$ & $0.9 \pm 0.4$ & $0.9 \pm 0.4$ & $2.9 \pm 0.8$ \\
\hline Fibroblasts/fibrocytes $^{\mathrm{a}}$ & PLGA/CaP & $0.0 \pm 0.0$ & $0.1 \pm 0.4$ & $0.0 \pm 0.0$ & $0.1 \pm 0.4$ \\
\hline Foreign body giant cells $^{\mathrm{b}}$ & PLGA/Ag-CaP & $0.0 \pm 0.0$ & $0.0 \pm 0.0$ & $0.0 \pm 0.0$ & $0.0 \pm 0.0$ \\
\hline Macrophages circles $^{\mathrm{b}}$ & PLGA/CaP & $0.0 \pm 0.0$ & $0.3 \pm 0.5$ & $0.3 \pm 0.5$ & $0.6 \pm 0.8$ \\
& PLGA/Ag-CaP & $0.3 \pm 0.5$ & $0.5 \pm 0.5$ & $0.3 \pm 0.5$ & $1.0 \pm 0.9$ \\
\hline Bone islands $^{\mathrm{b}}$ & PLGA/CaP & $0.3 \pm 0.5$ & $0.9 \pm 0.4$ & $0.7 \pm 0.5$ & $1.9 \pm 0.9$ \\
\hline Osteoclasts $^{\mathrm{b}}$ & PLGA/Ag-CaP & $0.6 \pm 0.7$ & $0.8 \pm 0.7$ & $0.8 \pm 0.5$ & $2.1 \pm 1.5$ \\
\hline & PLGA/CaP & $0.3 \pm 0.5$ & $0.9 \pm 0.4$ & $0.4 \pm 0.5$ & $1.6 \pm 0.8$ \\
\hline
\end{tabular}

${ }^{a}$ Scores ( 0 for $0 \%, 1$ for $1-25 \%, 2$ for $26-50 \%, 3$ for $>50 \%$ ) for percentage fraction of the cells per power field and the corresponding sum for each cell type.

${ }^{\mathrm{b}} \mathrm{S}$ cores $(0$ for none, 1 for $1-5,2$ for $6-10,3$ for $>11)$ for the absolute number of the cells per power field and the corresponding sum for each cell type.

efficacy were included in order to not complicate the overall cellular picture.

\section{Bone Formation}

The highly porous scaffolds enabled efficient cell ingrowth, which facilitated new bone formation everywhere in the former defect and additionally led to resorption of the biodegradable polymer fibres. Even within 8 weeks, bone formation was observed from the edge to the centre of the original defect (Fig. 6b, d). The fibres provided the necessary mechanical stability for mineralization and formation of cancellous bone. The necessity of filling drill hole defects with a bone graft has been shown in a recent study [33]. Defects left empty in the same defect model in sheep allowed only slow bone formation from the border of the former defects towards the centre: Histological investigations after two months implantation showed adipose cells in between newly formed bone and an organized blood clot in the centre of the defect (Fig. 6a). With increasing implantation time, the blood clot was resorbed and replaced by adipose and fibrous tissue containing predominantly fibrocytes. This resulted in bone resorption after four months implantation (Fig. 6c). The percentages of new bone matrix for empty defects increased from the $2^{\text {nd }}$ month $(43.2 \%)$ to the $4^{\text {th }}$ month $(58.6 \%)$ but decreased then to $27.3 \%$ after six months implantation (Table 2). PLGA/CaP and PLGA/Ag$\mathrm{CaP}$ treated defects showed newly formed bone after two months implantation with an area fraction of $20.5 \%$ and $22.5 \%$, respectively. However, bone formation is expected to continue throughout the defect after two months implantation as there were no signs of cellular reactions, which would influence the bone healing negatively. Moreover, histological evaluation showed regions with tissue that will induce shortly endochondral ossification and will become bone tissue.

\section{Comparison of Bone Cotton Wool to Other Bone Substitutes}

The use of this cotton wool-like biomaterial is beneficial for bone regeneration compared to other rigid implant materials. In a recent study by Schneider et al. [30], PLGA/CaP 60:40 nanocomposites were used to treat circular defects (diameter $=6 \mathrm{~mm}$ ) in the cranium of New Zealand White rabbits. There, the percentage of newly formed bone after four weeks implantation was significantly improved for $\mathrm{CaP}$ containing fibres $(34.9 \pm 17.0 \%)$ if compared to pure PLGA fibres $(25.1 \pm 14.6 \%)$. Therefore, pure PLGA fibres were not included within the present study. The bone regeneration was more advanced after four weeks implantation in the rabbit than after eight weeks implantation in sheep, demonstrating that bone formation is difficult to compare in between different defect models and animals. For this reason, the performance of the bone cotton wool-like material is compared with other artificial bone substitutes in the same defect model in sheep. Commercially available and injectable calcium phosphate cements assessed in sheep for two months resulted in similar area fractions of new bone (18.0\% for Norian $\mathrm{SRS}^{\circledR}$ and $24.8 \%$ for chronOS Inject ${ }^{\mathrm{TM}}$ ) [33] as obtained with the PLGA/CaP nanocomposites (Table 2). However, even though the brushite cement (chronOS Inject $^{\mathrm{TM}}$ ) resorbed faster than the apatite cement (Norian SRS $^{\circledR}$ ), new bone formation was not observed in the centre of the defect and was controlled through the resorption front. The resorption of the brushite cement occurred mainly through macrophages in a similar way as observed for the PLGA/CaP nanocomposites. Porous $\beta$-TCP blocks [34] investigated in the same sheep model showed within the first six weeks implantation faster resorption of the material than formation of new bone (12.8\% for blocks with pore diameter of $260 \mu \mathrm{m}$ ). Only few newly formed bone tissue was histomorphometrically observed in the centre of the 


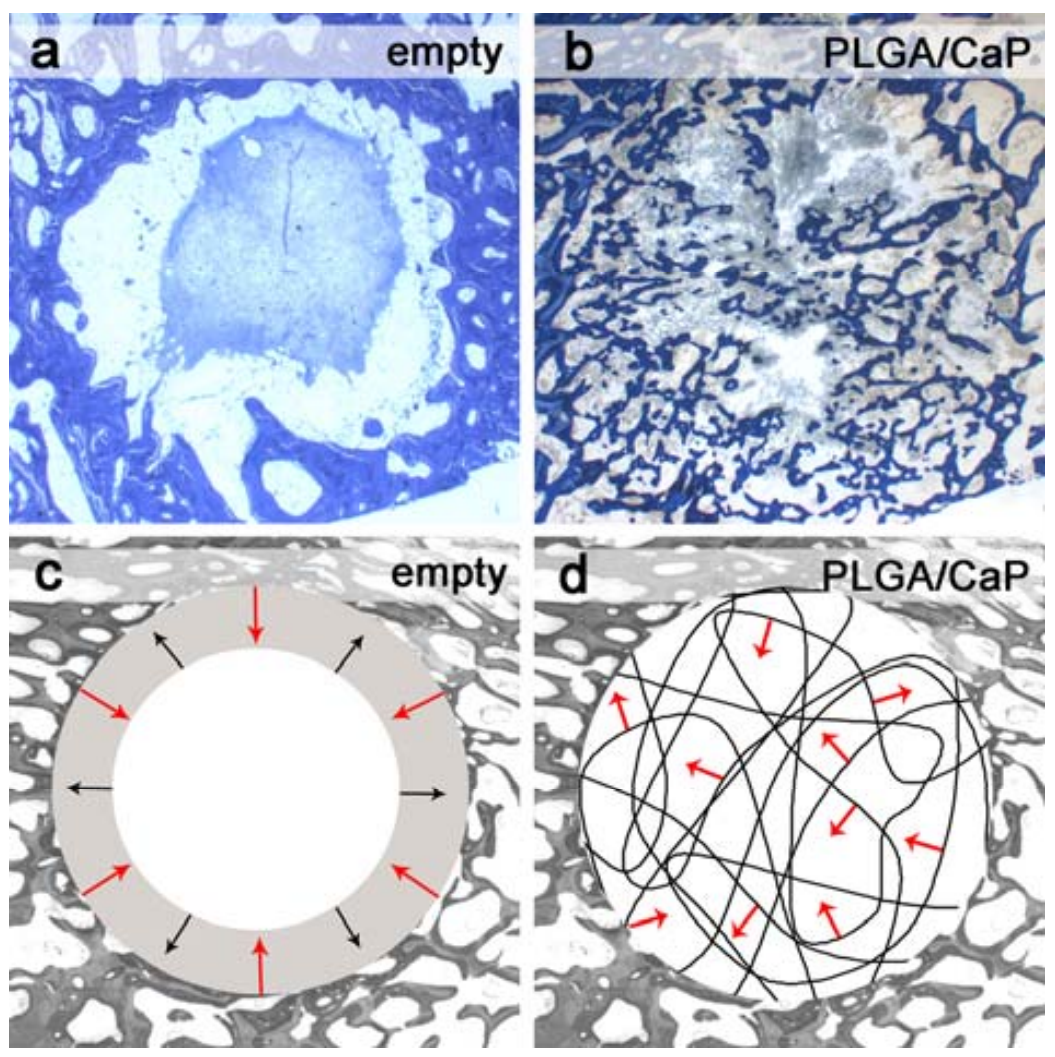

Fig. (6). Bone formation in defects left empty and for bone cotton wool treated defects. Histological ground sections for (a) empty defect shows the formation of an organized blood clot in the centre of defect whereas for (b) PLGA/CaP treated defect bone formation (blue) is visible. Schematic description (c, d) of bone formation (red arrow) and bone resorption (black arrow).

defect, which may be attributed to the only partly available interconnected pores. However, the porous $\beta$-TCP blocks would enable mechanical loading in the defect right from the start, which can not be said for the here applied nanocomposites.

\section{CONCLUSIONS}

The easy applicable cotton wool-like material suggests application for non-load bearing complex shaped bone defects. The advantageous use of this compressible material would be foreseen generally to be valuable in minimally invasive surgery or for specific indications in dental surgery (i.e. sinus augmentation, augmentation procedures of the alveolar bone, socket preservation after tooth extraction) where the antimicrobial properties of PLGA/Ag-CaP could be beneficial. Before testing this cotton wool-like material in humans, further investigations in animals are necessary to evaluate bone regeneration over a longer time period.

\section{ACKNOWLEDGEMENT}

Financial support by ETH Zurich is kindly acknowledged.

Table 2. Area Fraction of Newly Formed Bone After Implantation of Different Materials in Created Circular (Diameter = 8 mm, Depth $=13 \mathrm{~mm}$ ) Defects in Long Bone of Sheep

\begin{tabular}{|c|c|c|}
\hline Material & Implantation Time/Months & Newly Formed Bone/\% \\
\hline PLGA/CaP 60:40 & 2 & 20.5 \\
\hline PLGA/Ag-CaP 60:40 & 2 & 22.5 \\
\hline Norian SRS a & 2 & 18.0 \\
\hline chronOS Inject $^{\circledR a}$ & 2 & 24.8 \\
\hline Porous $\beta$-TCP blocks ${ }^{\mathrm{b}}$ & 1.5 & 12.8 \\
\hline Defect left empty $^{\mathrm{a}}$ & 2 & 43.2 \\
\hline Defect left empty & 4 & 58.6 \\
\hline Defect left empty ${ }^{a}$ & 6 & 27.3 \\
\hline
\end{tabular}

${ }^{a}$ Results adopted from the study by Apelt et al. [33].

${ }^{b}$ Results adopted from the study by von Doernberg et al. [34]. 


\section{SUPPLEMENTARY INFORMATION}

This article contains supplementary material that can be viewed at publisher's website.

Suppl. material 1: Preparation and characterisation of cotton wool-like PLGA/CaP nanocomposites including flame spray synthesis of $\mathrm{CaP}$ nanoparticles.

Suppl. material 2: Schematic description of the investigated zones in the former defect for semi-quantitative histological evaluation.

Suppl. material 3: Microradiographs after eight weeks implantation.

Suppl. material 4: Semi-quantitative analysis of the cellular content after eight weeks implantation of bone cotton wool and applying a score system.

\section{REFERENCES}

[1] Burchardt H. The biology of bone-graft repair. Clin Orthop 1983; 174 : 28-42.

[2] Wimmer C, Krismer M, Gluch H, Ogon M, Stockl B. Autogenic versus allogenic bone grafts in anterior lumbar interbody fusion. Clin Orthop Rel Res 1999; 360: 122-6.

[3] Laurie SWS, Kaban LB, Mulliken JB, Murray JE. Donor-site morbidity after harvesting rib and iliac bone. Plast Reconstr Surg 1984; 73(6): 933-8.

[4] Lord CF, Gebhardt MC, Tomford WW, Mankin HJ. Infection in bone allografts - incidence, nature, and treatment. J Bone Joint Surg Am 1988; 70A(3): 369-76.

[5] Damien CJ, Parsons JR. Bone-graft and bone-graft substitutes - a review of current technology and applications. J Appl Biomater 1991; 2(3): 187-208.

[6] Williams RL, Doherty PJ, Vince DG, Grashoff GJ, Williams DF. The biocompatibility of silver. Crit Rev Biocompat 1989; 5(3): 221-43.

[7] Jain J, Arora S, Rajwade JM, et al. Silver nanoparticles in therapeutics: development of an antimicrobial gel formulation for topical use. Mol Pharm 2009; 6(5): 1388-401.

[8] Hong KH. Preparation and properties of electrospun poly (vinyl alcohol)/silver fiber web as wound dressings. Polym Eng Sci 2007; 47(1): 43-9.

[9] Cavalu S, Simona V, Albona C, Hozan C. Bioactivity evaluation of new silver doped bone cement for prosthetic surgery. J Optoelectron Adv Mater 2007; 9(3): 690-3.

[10] Dorozhkin SV, Epple M. Biological and medical significance of calcium phosphates. Angew Chem-Int Edit 2002; 41(17): 3130-46.

[11] Hench LL. Bioceramics. J Am Ceram Soc 1998; 81(7): 1705-28.

[12] Kokubo T, Kim HM, Kawashita M. Novel bioactive materials with different mechanical properties. Biomaterials 2003; 24(13): 2161-75.

[13] Rezwan K, Chen QZ, Blaker JJ, Boccaccini AR. Biodegradable and bioactive porous polymer/inorganic composite scaffolds for bone tissue engineering. Biomaterials 2006; 27(18): 3413-31.

[14] Stevens MM. Biomaterials for bone tissue engineering. Mater Today 2008; 11(5): 18-25.

[15] Middleton JC, Tipton AJ. Synthetic biodegradable polymers as orthopedic devices. Biomaterials 2000; 21(23): 2335-46.

[16] Reneker DH, Chun I. Nanometre diameter fibres of polymer, produced by electrospinning. Nanotechnology 1996; 7(3): 216-23.

[17] Simonet M, Schneider OD, Neuenschwander P, Stark WJ. Ultraporous 3D polymer meshes by low-temperature electrospinning: Use of ice crystals as a removable void template. Polym Eng Sci 2007; 47(12): 2020-6.
[18] Hutmacher DW. Scaffolds in tissue engineering bone and cartilage. Biomaterials 2000; 21(24): 2529-43.

[19] Cai YZ, Wang LL, Cai HX, et al. Electrospun nanofibrous matrix improves the regeneration of dense cortical bone. J Biomed Mater Res Part A 2010; 95A(1): 49-57.

[20] Palin E, Liu HN, Webster TJ. Mimicking the nanofeatures of bone increases bone-forming cell adhesion and proliferation. Nanotechnology 2005; 16(9): 1828-35.

[21] Webster TJ, Ergun C, Doremus RH, Siegel RW, Bizios R. Enhanced functions of osteoblasts on nanophase ceramics. Biomaterials 2000; 21(17): 1803-10.

[22] Wei GB, Ma PX. Nanostructured biomaterials for regeneration. Adv Funct Mater 2008; 18(22): 3568-82.

[23] Zhang LJ, Webster TJ. Nanotechnology and nanomaterials: promises for improved tissue regeneration. Nano Today 2009; 4(1): 66-80.

[24] Misra SK, Mohn D, Brunner TJ, et al. Comparison of nanoscale and microscale bioactive glass on the properties of $\mathrm{P}(3 \mathrm{HB}) /$ Bioglass $(\mathrm{R})$ composites. Biomaterials 2008; 29(12): 1750-61.

[25] Meyer JL, Eanes ED. Thermodynamic analysis of secondary transition in spontaneous precipitation of calcium-phosphate. Calcif Tissue Res 1978; 25(3): 209-16.

[26] Meyer JL, Eanes ED. Thermodynamic analysis of amorphous to crystalline calcium-phosphate transformation. Calcif Tissue Res 1978; 25(1): 59-68.

[27] Loher S, Reboul V, Brunner TJ, et al. Improved degradation and bioactivity of amorphous aerosol derived tricalcium phosphate nanoparticles in poly(lactide-co-glycolide). Nanotechnology 2006; 17(8): 2054-61.

[28] Mohn D, Ege D, Feldman K, et al. Spherical calcium phosphate nanoparticle fillers allow polymer processing of bone fixation devices with high bioactivity. Polym Eng Sci 2010; 50(5): 952-60.

[29] Schneider OD, Loher S, Brunner TJ, et al. Cotton wool-like nanocomposite biomaterials prepared by electrospinning: In vitro bioactivity and osteogenic differentiation of human mesenchymal stem cells. J Biomed Mater Res Part B 2008; 84B(2): 350-62.

[30] Schneider OD, Weber F, Brunner TJ, et al. In vivo and in vitro evaluation of flexible, cotton wool-like nanocomposites as bone substitute material for complex defects. Acta Biomater 2009; 5(5): 1775-84.

[31] Schneider OD, Loher S, Brunner TJ, Schmidlin P, Stark WJ. Flexible, silver containing nanocomposites for the repair of bone defects: antimicrobial effect against E. coli infection and comparison to tetracycline containing scaffolds. J Mater Chem 2008; 18(23): 2679-84.

[32] Nuss KMR, Auer JA, Boos A, Von Rechenberg B. An animal model in sheep for biocompatibility testing of biomaterials in cancellous bones. BMC Musculoskelet Disord 2006; 7: 67.

[33] Apelt D, Theiss F, El-Warrak AO, et al. In vivo behaviour of three different injectable hydraulic calcium phosphate cements. Biomaterials 2004; 25(7-8): 1439-51.

[34] von Doernberg MC, von Rechenberg B, Bohner M, et al. In vivo behaviour of calcium phosphate scaffolds with four different pore sizes. Biomaterials 2006; 27(30): 5186-98.

[35] Loher S, Stark WJ, Maciejewski M, et al. Fluoro-apatite and calcium phosphate nanoparticles by flame synthesis. Chem Mater 2005; 17(1): 36-42.

[36] Pratsinis SE. Flame aerosol synthesis of ceramic powders. Prog Energy Combust Sci 1998; 24(3): 197-219.

[37] Stark WJ, Pratsinis SE, Maciejewski M, Loher S, Baiker A, inventors; Flame synthesis of metal salt nanoparticles, in particular calcium and phosphate comprising nanoparticles. WO patent 087660 A1. 2005.

[38] Loher S, Schneider OD, Maienfisch T, Bokorny S, Stark WJ. Microorganism-triggered release of silver nanoparticles from biodegradable oxide carriers allows preparation of self-sterilizing polymer surfaces. Small 2008; 4(6): 824-32.

[39] Nunamaker DM. Experimental models of fracture repair. Clin Orthop 1998; 355: S56-S65. 